\title{
LA JUDICIARISATION DE LA POLITIQUE, LES DROITS DES MINORITES ET LE NATIONALISME CANADIEN*
}

\author{
Linda Cardinal
}

Ce texte propose un commentaire sur les rapports entre le droit et la politique à partir de trois cas de figure : le phénomène de la judiciarisation de la politique, la question des droits des minorités linguistiques, et le nationalisme canadien. Trois thèmes qui méritent un développement à part, mais qu'il faut aussi tenter d'analyser comme des éléments d'un tout car ils sont liés, bien que de façon contradictoire. En effet, si la judiciarisation de la politique a favorisé le développement des droits linguistiques au Canada et la démocratisation de la justice, elle a aussi coïncidé avec l'avènement d'un nationalisme des droits fondé exclusivement sur la référence à des droits individuels pancanadiens, une idéologie qui rend difficile l'accommodement entre les groupes. Dit autrement, nous avons été témoin, au Canada, de l'avènement d'un populisme des droits qui, paradoxalement, a favorisé le rétrécissement de l'espace politique canadien au profit d'un mouvement de réconciliation de la nation avec elle-même heurtant de front l'idée d'une ouverture à l'Autre qu'a rendu possible une certaine tradition canadienne fondée sur le binationalisme. Pour dire les choses encore plus brusquement, obsédé par son unité, le Canada a cherché de plus en plus à fusionner avec lui-même. Il a fondé son identité sur un nationalisme des droits, un nationalisme apparemment civique, sauf que celui-ci a, jusqu'à présent, fait peu de place au débat démocratique, notamment au débat sur les institutions politiques et à l'aménagement de rapports plus justes entre les groupes nationaux et ce, malgré une représentation de soi fondée sur la diversité.

\section{LA JUDICIARISATION DE LA POLITIQUE}

L'on a beaucoup péroré sur le thème de la judiciarisation de la politique au Canada. À la différence de la plupart des commentateurs de la question, David Smith, dans son remarquable ouvrage, The Invisible Crown. The First Principle of Canadian Government ${ }^{1}$ - malheureusement passé inaperçu en milieu francophone - a souligné que le mouvement, tel que nous le connaissons aujourd'hui, dans toute son ampleur, ne commence pas en 1982 avec l'adoption de la Charte $^{2}$, mais plutôt avec la mise sur pied du Programme de contestation judiciaire ${ }^{3}$. Nous avons aussi tenté de documenter davantage la question en montrant le lien intime entre le phénomène de la judiciarisation de la politique, le Programme et le rôle clé de l'exécutif dans la promotion de la logique des droits ${ }^{4}$. Ainsi, dès les années soixante-dix, la judiciarisation est voulue, du moins souhaitée par l'exécutif afin de mieux lutter contre les provinces et d'accroître la légitimité du gouvernement fédéral comme unique lieu d'identification à une nation juridique fondée sur des valeurs définies dorénavant par des Juges

* Ce texte a été possible grâce à une subvention du CRSH sur le thème de la culture politique des droits au Canada.

Toronto, University of Toronto Press, 1995.

2 Charte canadienne des droits et libertés, partie I de la Loi constitutionnelle de 1982, constituant l'annexe B de la Loi de 1982 sur le Canada (R.-U.), 1982, c. 11 [Charte].

3 Le Programme de contestation judiciaire [Programme], lancé en 1978, à été créé par l'exécutif au but de clarifier, grâce au financement de causes types d'importance nationale, les dispositions constitutionnelles relatives aux droits à l'égalité et aux droits linguistiques. Voir généralement en ligne : Programme de contestation judiciaire du Canada $<$ http://www.ccppcj.ca $>$.

4 Linda Cardinal, «Le pouvoir exécutif et la judiciarisation de la politique au Canada. Une étude du Programme de contestation judiciaire» (2000) 20:1 Politique et Sociétés 43. 
au lieu de faire appel aux élus ou aux citoyens pour en débattre démocratiquement au sein de l'espace public.

David Smith considère que la judiciarisation de la politique au Canada est venue principalement d'une prise de conscience des limites du pouvoir exécutif à l'égard des provinces canadiennes. Selon Smith, plus les provinces jouent un rôle dans la gestion des affaires de la fédération canadienne et adopter leurs lois, plus le gouvernement fédéral comprend qu'il ne peut plus comme au XIX ${ }^{\mathrm{e}}$ siècle, se prévaloir de son pouvoir de désaveu à leur égard de même qu'il ne peut uniquement s'en tenir à demander des avis au judiciaire dans ses conflits avec les gouvernements provinciaux. De toute façon, dès la fondation du Canada, il est tenu pour acquis que le gouvernement fédéral n'utilisera pas son pouvoir de désaveu dans le domaine des questions d'ordre culturelles, linguistiques ou religieuses ${ }^{5}$.

De plus, pendant les années 1960 et 1970, la question linguistique, la montée du néonationalisme au Québec et du Parti québécois viennent accentuer les difficultés du gouvernement fédéral, notamment à l'égard du Québec. L'adoption de la Charte de la langue française du Québec en $1977^{6}$ — la Loi 101 — consacrant le français, langue de travail ainsi que langue officielle du Québec en matière législative et judiciaire constitue un événement déterminant au pays. Force est de constater que c'est à partir de ce moment que le gouvernement décide de la mise sur pied du Programme. Il choisit expressément la voie de la judiciarisation au détriment d'une autre voie possible tout à fait compatible avec les traditions constitutionnelles canadiennes, notamment celle de la négociation ou du compromis politique.

Ces quelques éléments de contexte révèlent qu'à partir des années 1970, le gouvernement fédéral, plus précisément l'exécutif, cherche à se donner de nouveaux moyens dans le règlement de ses conflits avec les provinces, notamment en qui

Pour plus de détails sur la question du pouvoir de désaveu, voir le remarquable ouvrage de Robert Vipond, Liberty and Community: The Failure of the Canadian Constitution, Albany, The University of New York University Press, 1991.

6 L.R.Q. 1977, c. C-11. a trait à la question linguistique. Dans ce domaine, les références plus traditionnelles à l'idée de convention, de compromis historique et à la possibilité de négociations ne représentent pas des voies de solution pour l'exécutif. La mise en place du Programme constitue le meilleur moyen de régler le conflit sur la langue et les minorités linguistiques en le déplaçant vers les tribunaux. Soulignons également que ces derniers sont amenés à se prononcer sur une foule d'autres questions dans le domaine identitaire. En effet, à la même époque, soit en 1986, les Juges ont eu à décidé du contenu des valeurs canadiennes dans l'arrêt Oakes $^{7}$ alors qu'un tel débat aurait aussi pu avoir lieu dans l'enceinte du Parlement ou avec les citoyens. Pour revenir au Programme de contestation judiciaire, même s'il réalise un travail remarquable au plan de la démocratisation de la justice au Canada, notamment après 1985, notre hypothèse est qu'il est aussi au service des aspirations de l'exécutif d'amener les Juges à poser des jugements sur des questions d'ordre politique comme la question linguistique afin de mettre fin une fois pour toute au débat sur la langue. L'exécutif tire ainsi profit d'une telle stratégie car sa légitimité se voit renforcée comme principal gardien des valeurs canadiennes. Or, qu'en est-il des effets pervers de la judiciarisation de la politique dans le domaine des droits linguistique? Dit autrement, le gouvernement fédéral a t-il réussi à rendre plus acceptable les droits des minorités linguistiques, notamment ceux des francophones vivant à l'extérieur du Québec en favorisant leur judiciarisation?

\section{LES DROITS DES MINORITÉS LINGUISTIQUES}

À bien des égards, la judiciarisation des droits a fait faire un bond important aux minorités de langue officielle au Canada, notamment en leur octroyant le droit de gérer leurs écoles. De façon plus générale, celle-ci a aussi servi à faire avancer la théorie judiciaire des droits linguistiques qui reconnaît l'importance d'accorder une forme de droits collectifs aux minorités de langue officielle. Ainsi, les tribunaux ont réussi à faire accepter le besoin de mesures spéciales à l'intention des

\footnotetext{
R. c. Oakes, [1986] 1 R.C.S. 103, en ligne: IIJCan $<$ http://www.canlii.org/ca/jug/csc/1986/1986csc7.html>.
} 
minorités de langue officielle au Canada. Dans les arrêts Beaulac ${ }^{8}$ et Arsenault-Cameron ${ }^{9}$, les Juges ont posé les jalons d'une théorie judiciaire des droits collectifs qui somme les législateurs de tenir compte des objectifs de la loi sur les langues officielles lorsqu'ils adoptent des mesures à l'intention de leurs citoyens, notamment celui de voir au développement et à l'épanouissement des minorités de langue officielle.

Toutefois, le discours sur les droits linguistiques a aussi pris une orientation contradictoire. Dit autrement, celui-ci génère des effets pervers. À titre d'exemple, l'idée de voir dans les droits linguistiques un droit fondamental a servi à conforter l'avènement d'un populisme juridique. De plus, à chaque fois que les minorités linguistiques choisissent de porter une cause devant les tribunaux, c'est afin de s'assurer d'une reconnaissance constitutionnelle de leurs droits linguistiques sans toujours, par ailleurs, se demander dans quelle mesure ces nouvelles reconnaissances vont augmenter leur capacité d'action ou d'intervention.

De façon plus explicite, l'idée selon laquelle la langue constitue un droit fondamental n'est pas nouvelle. Celle-ci est déjà énoncée, en 1948, dans la Déclaration universelle des droits de l'Homme ${ }^{10}$ des Nations Unies. Les plus récents instruments dont s'est dotée la Communauté européenne dont la Charte européenne des langues régionales ou minoritaires $^{11}$ et la Convention-cadre pour la protection des minorités nationales reconnaissent également les droits linguistiques comme des droits de la personne. Toutefois, ces derniers fondent aussi le développement des politiques linguistiques sur une idée de diversité au lieu de la logique des droits.

$\mathrm{Au}$ Canada, la conception des droits linguistiques comme des droits de la personne est non seulement en conformité avec l'évolution des droits fondamentaux et des droits linguistiques au

\footnotetext{
R. c. Beaulac, [1999] 1 R.C.S. 768, en linge: IIJCan <http://www.canlii.org/ca/jug/csc/1999/1999csc26.html>. Arsenault-Cameron c. Île-du-Prince Édouard, [2000] 1 R.C.S. 3 , 2000 CSC 1, en ligne: IIJCan $<$ http://www.canlii.org/ca/jug/csc/2000/2000csc1.html $>$.

10 Rés. AG 217 (III), Doc. Off. AG NU, $3^{\mathrm{e}}$ sess., supp. nº 13, Doc. NU A/180 (1948).

115 novembre 1992, Eur. T.S. $1992 \mathrm{n}^{\mathrm{o}} 148$ (entrée en vigueur : $1^{\mathrm{er}}$ mars 1998).
}

plan international. Les droits à la liberté d'expression et le droit à l'égalité y ont pris une importance nouvelle que l'on a exploitée largement dans le cas de la mise en cause de certaines dispositions de la Loi 101 dont celles portant sur l'affichage commercial et les raisons sociales au Québec. Le discours d'Alliance Québec dans ce domaine est révélateur de cette orientation plus individualiste conférée aux droits linguistiques comme droits fondamentaux. Faut-il le rappeler, un groupe comme Alliance Québec revendique vigoureusement l'égalité absolue des langues. Celui-ci exige le bilinguisme au Québec, notamment une offre active de services en anglais à la fois de la part du gouvernement du Québec et $\mathrm{du}$ Canada et non uniquement une sorte d'obligation d'accommodement de la langue de la minorité. Les minorités francophones hors Québec réclament aussi une offre active de services en français dans leur province respective mais leurs succès sont plus mitigés. Ainsi, au Canada, la référence aux droits fondamentaux a contribué à transformer le discours sur les droits linguistiques en une sorte de principe a-historique, transcendant fondée sur une référence à la langue comme un principe absolu.

Ces quelques éléments de réflexion rejoignent bien l'idée de Peter Russell selon laquelle la Charte a rendu possible une telle conscience civique des droits dont l'effet est de concevoir toute référence aux droits comme un droit absolu $^{12}$. Dans un texte sur le populisme et la politique des droits, Knopff s'en est aussi pris à la façon dont la judiciarisation a encouragé les extrémismes au plan politique au lieu de favoriser la modération ${ }^{13}$. Tant Russell que Knopff citent le débat sur l'affichage au Québec comme un cas de figure d'extrémisme. Toutefois, cela ne fait pas de ces derniers des défenseurs de la Loi 101 que Knopff qualifie plutôt de «foolish law ${ }^{14}$.

Au plan historique, l'idée selon laquelle la fédération canadienne reposait sur un compromis historique devait permettre de refléter l'esprit du fédéralisme, notamment dans le domaine des

\footnotetext{
12 «Canadian Constraints on Judicialisation From Without» (1994) 15:2 International Journal of Political Science 165.

13 Rainer Knopff, «Populis and the Political Right: The Dual Attack on Representative Democracy» (1998) 31:4 Revue canadienne de science politique 683, à la p. 702 .

14 Ibid.
} 
droits des minorités. Le thème du compromis servait aussi à reconnaître le principe de l'autonomie des provinces. Il visait à permettre aux provinces d'évoluer à leur propre rythme. Or, est-ce que les minorités linguistiques s'en portent mieux depuis qu'Alliance Québec fait la guerre au Québec et que les minorités francophones hors Québec ont également opté pour la voie judiciaire? Le jugement de la Cour supérieure de justice de l'Ontario annulant la décision de la Commission de restructuration des services de santé en Ontario concernant la fermeture de l'hôpital Montfort a affirmé que la minorité franco-ontarienne demeure une communauté menacée. ${ }^{15}$ Le Sénateur Jean Maurice Simard a indiqué, dans son rapport sur la situation des minorités francophones hors Québec, que leur développement n'était toujours pas assuré et que l'État canadien devait faire mieux ${ }^{16}$. Quant à la minorité anglo-québécoise, elle a fort bien réussi à compliquer davantage son rapport avec la majorité francophone du Québec depuis les années 1970 et la situation est loin d'être radieuse. Voilà, il me semble, quelques effets pervers découlant du recours au judiciaire qui ne sont malheureusement pas pris en compte dans le débat sur l'avancement ou le développement des minorités francophones hors Québec. L'absolutisation des droits linguistiques ne fait pas écho à un plus grand dynamisme en milieu minoritaire.

\section{DROITS ET NATIONALISME}

Une des conséquences de la judiciarisation de la politique a également été de permettre le renouveau du nationalisme canadien. Comme nous l'avons vu plus haut, alors que la question des droits linguistiques est de plus en plus associée à une question de droits fondamentaux dorénavant compris comme des droits individuels absolus, force est de constater que ce discours n'est pas sans lien avec le développement d'un nationalisme pancanadien fondée sur l'idée que l'anglais doit être prédominant partout au Canada. Will Kymlicka a bien identifié le phénomène dans un

15 Lalonde c. Ontario (Commission de restructuration des services de santé) (2001), 56 O.R. (3 $) 505$ (CA), en ligne: IIJCan <www.canlii.org/on/cas/onca/2001/2001 onca10003. html\#sum>.

16 Jean-Maurice Simard, De la coupe aux lèvres : un coup de coéur se fait attendre, Ottawa, Le senate de Canada, 1999. texte qu'il a publié dans le cadre du projet Sortir de l'impasse ${ }^{17}$. Ce nationalisme a une dimension ethnique alors qu'il se présente comme le représentant de l'universel.

Que l'on pense à la lutte acharnée d'Alliance Québec contre la Loi 101 ou au discours de l'ancien Reform Party contre les droits des peuples des Premières Nations ou encore contre la reconnaissance du Québec comme société distincte, la référence à des droits individuels a été transformée en une sorte de credo servant à lutter contre toute revendication de droits collectifs, cette dernière étant associée à du particularisme ou encore à de l'ethnicisme. Or, pendant ce temps, le nationalisme juridique réussi essentiellement à réduire l'espace de la communauté à l'identité d'un groupe en particulier, celle des Canadiens anglais de souche et ceux qui s'identifient à eux. À une autre époque, nous aurions parlé d'un mouvement visant 1'anglo-conformité. Claude Couture a proposé la notion de « libéralisme ethnique » pour traduire essentiellement la même idée $^{18}$. De façon plus générale, le discours des droits individuels vient conforter les Canadiens anglais dans leur position de groupe dominant, un groupe qui incarnerait spontanément l'universel comme si ce dernier pouvait être le propre d'une culture donnée. Dans le nationalisme canadienanglais, l'universalisme ne vient pas de la distance critique que l'on peut exercer à l'égard de son milieu. L'universel, étrangement, vient du refus de la différence à moins qu'elle ne soit pas menaçante, une différence de façade comme dans un certain folklore canadien-français ou dans un certain multiculturalisme qui sert à renforcer le nationalisme canadien plutôt qu'à revendiquer une meilleure prise en compte des préoccupations des immigrants. Ce nationalisme rend aussi impossible dans le fédéralisme de penser la question de la différence de façon significative. Il contribue au pourrissement du débat Québec-Canada tout comme il n'aide pas la reconnaissance plus grande des peuples des Premières Nations ou des minorités culturelles et linguistiques.

\footnotetext{
7 «Le fédéralisme multinational au Canada : un partenariat à repenser» dans Guy Laforest et Roger Gibbins, dir., Sortir de l'impasse. Les voies de la réconciliation, Montréal, IRPP, 1998, 15.

18 Paddling with the current : Pierre Elliott Trudeau, Etienne Parent, liberalism, and nationalism in Canada, Edmonton, The University of Alberta Press, 1998, à la p. 9.
} 
Pour terminer, force est malheureusement de constater qu'il n'existe pas de volonté politique au Canada qui pourrait donner lieu à une réforme constitutionnelle favorable à une plus grande ouverture à l'identité ou aux droits collectifs. L'époque de la politique de l'identité est probablement révolue et l'on ne doit pas s'attendre à une trop grande ouverture de la part des gouvernements fédéral et provinciaux à l'égard du Québec ou des groupes minoritaires. Dans le contexte de la cause Montfort l'attitude du gouvernement ontarien à l'égard des francophones de la province a montré que la politique de l'identité est belle et bien chose du passé.

Certes, il y a lieu de réformer le fédéralisme en vue d'une plus grande transparence du processus de formulation des politiques, d'une plus grande imputabilité de la part du gouvernement à l'égard de la population, d'un meilleur équilibre entre l'idée de normes nationales et les préoccupations des provinces. Toutefois, les récentes avancées de la thèse du fédéralisme asymétrique montrent bien les difficultés que pose au Canada anglophone, surtout en Ontario comme dans l'ouest du pays, d'accommoder différentes conceptions de la nation.

\section{Linda Cardinal}

Chaire de recherche sur la francophonie et les politiques publiques

École d'études politiques, Université d'Ottawa 